\title{
Differentiation of mucinous from non-mucinous pancreatic cyst fluid using dual-stained, 1 dimensional polyacrylamide gel electrophoresis
}

\author{
John M Streitz ${ }^{1 *}$, Michael T Madden², Wilmar Salo ${ }^{2}$, Kirk P Bernadino ${ }^{4}$, Joseph L Deutsch ${ }^{3}$ and John C Deutsch ${ }^{4}$
}

\begin{abstract}
Background: Pancreatic cysts are being increasingly identified in patients. Mucinous cysts have malignant potential whereas non-mucinous cysts do not. Distinguishing potentially malignant cysts from harmless ones by the characterization of cyst fluid contents remains a difficult problem. This study was undertaken to determine whether cyst fluid mucin glycoprotein analysis could differentiate mucinous from non-mucinous pancreatic cysts.

Methods: Cyst fluid from 28 patients who underwent resection of a pancreatic cyst was used for the study. In each case the type of cyst was histologically identified. One dimensional SDS polyacrylamide gel electrophoresis (1D-SDS PAGE) was performed on cyst fluid samples. For the detection of the separated proteins, we employed a novel dual staining technique. The gel was first stained with periodic acid Schiff (PAS), a mucin histochemical stain followed by a secondary protein staining with Simply Blue Safestain (Invitrogen).

Results: Visual scoring (based on the presence of mucins) gave a sensitivity of 95\%, a specificity of 100\%, a positive predictive value of 100\%, and a negative predictive value of $88 \%$ for prediction of mucinous histology.

Conclusions: One dimensional SDS polyacrylamide gel electrophoresis of pancreatic cyst fluid, followed by mucin (PAS) and protein (Simply Blue Safestain) staining, provides a means of concentrating and visualizing mucins, which allows the accurate differentiation of mucinous from non-mucinous histology in pancreatic cysts.
\end{abstract}

Keywords: Pancreatic cancer, Pancreatic cyst fluid, Biomarkers, Mucins, 1-D-PAGE

\section{Background}

Pancreatic cysts are being increasingly identified by improved imaging methods, including endoscopic ultrasound (EUS), which allows cyst fluid sampling. Some mucinous cysts have malignant potential, whereas non-mucinous cysts do not. Surgical resection is often recommended for mucinous cystic lesions of the pancreas because of cancer risk [1,2], but differentiating harmless cysts from potentially malignant ones remains difficult. Whether to resect or observe certain cysts is a common clinical problem owing to the inability to distinguish mucinous from nonmucinous cysts.

Pancreatic neoplasms with malignant potential such as mucinous cystadenoma, and intraductal papillary mucinous

\footnotetext{
*Correspondence: john.streitz@essentiahealth.org

'Departments of Surgery, Essentia Health System/Duluth Clinic; University of Minnesota Medical School, Duluth, MN, USA

Full list of author information is available at the end of the article
}

neoplasm (IPMN), can be distinguished to some extent, using cyst fluid analysis techniques, from cysts with no malignant potential, such as serous cystadenoma and pseudocyst. Cytology of cyst fluid showing dysplastic features is strongly predictive of mucinous neoplasia but is often falsely negative [3]. Clinical staining methods looking for mucin in the cyst fluid of pancreatic cysts have shown variable positivity but are considered unreliable $[4,5]$. The cyst fluid carcinoembryeonic antigen (CEA) level has proven more reliable but is not a consistent positive predictor of mucinous histology by itself [6].

This research was undertaken to evaluate a pancreas cyst fluid characteristic that could reliably identify mucinous histology and therefore help to guide therapy. Mucinous cystic lesions of the pancreas are known to express mucins in their cyst fluid [7]. Clinical mucin staining often fails to identify mucins, either because of dilution, low level of expression, inhomogeneous distribution within the fluid 
sample or interference by other glycoproteins. Research has shown that mucins are uniformly found in the high molecular weight region ( $>250 \mathrm{~K}$ daltons) of a one dimensional, polyacrylamide gel $[8,9]$. Using the technique of one dimensional, polyacrylamide gel electrophoresis (1D-SDS PAGE) we were able to separate mucins from smaller proteins within the fluid, concentrating mucins near the origin of the gel. The dual staining procedure effectively demonstrated the presence of mucins in the cyst fluid of mucinous cystic lesions.

\section{Results}

Results of the stain scoring are shown in Table 1 . None of the non-mucinous cysts showed staining for mucin, whereas all of the mucinous cystadenomas and 14 of the 15 IPMN specimens (93\%) showed positive mucin staining. As a test for detecting mucinous neoplasms, these results yielded a sensitivity of $95 \%$, a specificity of $100 \%$, a positive predictive value of $100 \%$, and a negative predictive value of $88 \%$. Prediction intervals using a binomial model were calculated for the number of false negatives and false positives; under this model each count will be less than $19 \%$ of the sample size with a probability of 0.95 .

CEA Results (reported as $\mathrm{ng} / \mathrm{ml}$ ) are listed in Table 2. Using a CEA level of $>200 \mathrm{ng} / \mathrm{ml}$ to indicate mucinous histology [10], CEA results had one false positive and two false negative results.

Four samples did not reach the 5 ug level for protein loading. All of these were mucinous samples and had total protein content of $1.95 \mathrm{ug}, 1.6 \mathrm{ug}, 1.6 \mathrm{ug}$ and 3.2 ug; they were all scored as positive for mucin, with respective protein scores of $1,2,2$, and 2 .

\section{Discussion}

Diagnostic tests of pancreatic cyst fluid that can accurately predict cyst wall histology are needed in order to differentiate between harmless cystic lesions and premalignant, mucinous lesions that would require surgical intervention. Mucicarmine fluid staining for extracellular mucin is a weak predictor of mucinous histology, with $38 \%$ to $55 \%$ of mucinous cystic lesions testing positive, as well as $40 \%$ of

Table 1 Histologic diagnosis compared to stain scoring

\begin{tabular}{lccc}
\hline & Total & \multicolumn{2}{c}{ Stain scoring } \\
\cline { 3 - 4 } Histologic diagnosis & Number & Negative & Positive \\
\hline Non-mucinous cyst & 3 & 3 & \\
Non-mucinous cyst & 4 & 4 & 5 \\
Pseudocyst & & & \\
Mucinous cyst & 6 & & 9 \\
IPMN benign & 9 & & 6 \\
IPMN malignant & 6 & & \\
Mucinous cystadenoma & & & \\
\hline
\end{tabular}

*False negative.
Table 2 Carcinoembryonic Antigen (CEA) levels, compared to staining scoring

\begin{tabular}{|c|c|c|}
\hline & & Mucin \\
\hline Histologic diagnosis & $\begin{array}{c}\text { CEA level } \\
(\mathrm{ng} / \mathrm{ml})\end{array}$ & Stain scoring \\
\hline \multicolumn{3}{|l|}{ Non-mucinous } \\
\hline P15 (cystic lymphangioma) & 0 & 0 - Negative \\
\hline Pan19 (serous microcystic adenona) & 0 & 0 - Negative \\
\hline P64 (lymphoepithelial cyst) & $1576^{* *}$ & 0 - Negative \\
\hline Pan18 (pseudocyst) & 0 & 0 - Negative \\
\hline Pan21 (pseudocyst) & 0 & 0 - Negative \\
\hline Pan22 (pseudocyst) & 0 & 0 - Negative \\
\hline P39 (pseudocyst) & 0 & 0 - Negative \\
\hline \multicolumn{3}{|l|}{ Mucinous } \\
\hline P10 (IPMN benign) & 994 & 3 - Positive \\
\hline P31 (IPMN benign) & $106^{*}$ & 2 - Positive \\
\hline P32 (IPMN benign) & 878 & 1 - Positive \\
\hline P43 (IPMN benign) & 1011 & 0 -Negative \\
\hline P46 (IPMN benign) & 589 & 2 - Positive \\
\hline P56 (IPMN benign) & 598 & 1 - Positive \\
\hline P4 (IPMN malignant) & 555 & 2 - Positive \\
\hline P7 (IPMN malignant) & 438 & 1 - Positive \\
\hline P41 (IPMN malignant) & 988 & 2 - Positive \\
\hline P55 (IPMN malignant) & 589 & 2 - Positive \\
\hline P57 (IPMN malignant) & 1493 & 3 - Positive \\
\hline P58 (IPMN malignant) & 654 & 2 - Positive \\
\hline P61 (IPMN malignant) & 229 & 2 - Positive \\
\hline P65 (IPMN malignant) & 329 & 1 - Positive \\
\hline P68 (IPMN malignant) & $26^{*}$ & 1 - Positive \\
\hline Pan16 (Mucinous cystadenoma) & 1572 & 1 - Positive \\
\hline P24 (Mucinous cystadenoma) & 517 & 2 - Positive \\
\hline P25 (Mucinous cystadenoma) & 2253 & 3 - Positive \\
\hline P40 (Mucinous cystadenoma) & 1065 & 3 - Positive \\
\hline P63 (Mucinous cystadenoma) & 208 & 2 - Positive \\
\hline P69 (Mucinous cystadenome) & 5 & 2 - Positive \\
\hline
\end{tabular}

*False negative; **False positive.

pseudocysts $[7,11]$. It has a reported positive predictive value of $61 \%$ in one study [6]. Cytology of cyst fluid is strongly predictive of mucinous histology when positive, but is an insensitive test. [6,10,12]. Cyst fluid CEA level has shown sensitivity when present in high amounts $[10,12]$, but its positive predictive value as an independent test in a large cooperative study was 79\% [10] and has been reported to be as low as $51 \%$ by others [6]. Furthermore, investigators use different levels of CEA to predict mucinous histology in different reports, depending on whether or not the goal is sensitivity, specificity or accurate. Exhaustive investigation into the entire cyst fluid 
Table 3 Postoperative histologic diagnosis

\begin{tabular}{lcc}
\hline Histologic diagnosis & Number & Percent \\
\hline Non-mucinous cyst & 7 & $25 \%$ \\
Serous microcystic adenoma & 1 & $3.6 \%$ \\
Cystic lymphangioma & 1 & $3.6 \%$ \\
Lymphoepithelial cyst & 1 & $3.6 \%$ \\
Pseudocyst & 4 & $14.3 \%$ \\
Mucinous cyst & 21 & $75 \%$ \\
Mucinous cystadenoma & 6 & $21.4 \%$ \\
IPMN benign & 6 & $21.4 \%$ \\
IPMN malignant & 9 & $34.2 \%$ \\
\hline
\end{tabular}

proteome using mass spectrometry has so far yielded no biomarkers any more useful than the CEA level $[5,13]$. 1-D SDS PAGE of pancreatic cyst fluid, utilizing a single protein specific stain (SYPRO Ruby fluorescence), has been employed by others with no demonstrable diagnostic value [8]. Specific cyst fluid (non-mucinous) glycoproteins have shown some promise as biomarkers for mucinous histology, with independent sensitivity in one study as high as $78 \%$ [8].

The 1-D SDS PAGE/mucin staining method we have described, shows a high sensitivity, specificity, positive predictive value, and negative predictive value for detecting mucinous neoplasms. This is due to the separation of mucin glycoproteins from other proteins and their concentration on the gel.

Protein electrophoresis is commonly employed in clinical laboratories. This makes it possible that this method could be converted to a diagnostic test for clinical use.
The visual scoring of mucin content is a weakness in the analysis. The use of densitometry or quantitation of the mucin glycoprotein levels might allow a more accurate evaluation. Nonetheless, visual scoring methods, such as the one that we employed, are often used in clinical medicine. The Her2 Neu staining for breast cancer, which is visually scored 0 to $3+$ based on an estimate of immunohistochemistry staining of cell membranes, is considered a reliable clinical tool. CEA levels continue to be the used as the primary clinical biomarker of mucinous cystic lesions. While CEA level was not part of our primary data analysis, the results are included as an interesting clinical correlation.

The expanding field of glycoproteomics makes use of mass spectrometry (MS) to analyze complex biologic fluids such as pancreatic cyst fluid. It requires the enrichment of the mucin glycoprotein content in the fluid to allow MS to identify peptides expressed in low concentrations. A number of methods have been used to achieve this enrichment, including lectin affinity chromatography, hydrophilic solid phase extraction, boric acid, and hydrazide chemistry [14,15]. The 1-D SDS PAGE/ dual staining technique presented herein displays the ability to effectively enrich the mucin glycoprotein in the samples, which should aid in further proteomic analyses of these cysts.

Our ongoing research to identify specific mucins present in pancreatic cyst fluid, using MS, could enable more exact differentiation between the various mucinous and non-mucinous cyst types. Particular mucins identified within the cyst fluid may prove to be useful as biomarkers for mucinous histology.

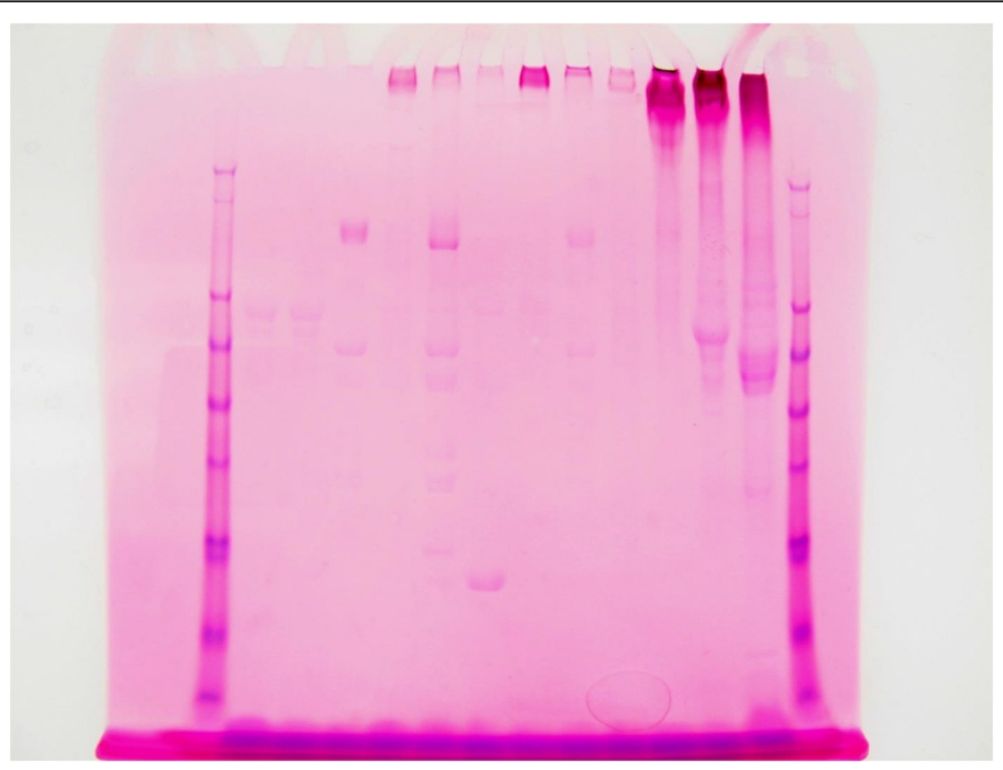

Figure 1 1-D SDS PAGE; primary PAS glycoprotein staining of representative pancreatic cyst fluid samples. (Direction of travel on the gel is downward). 


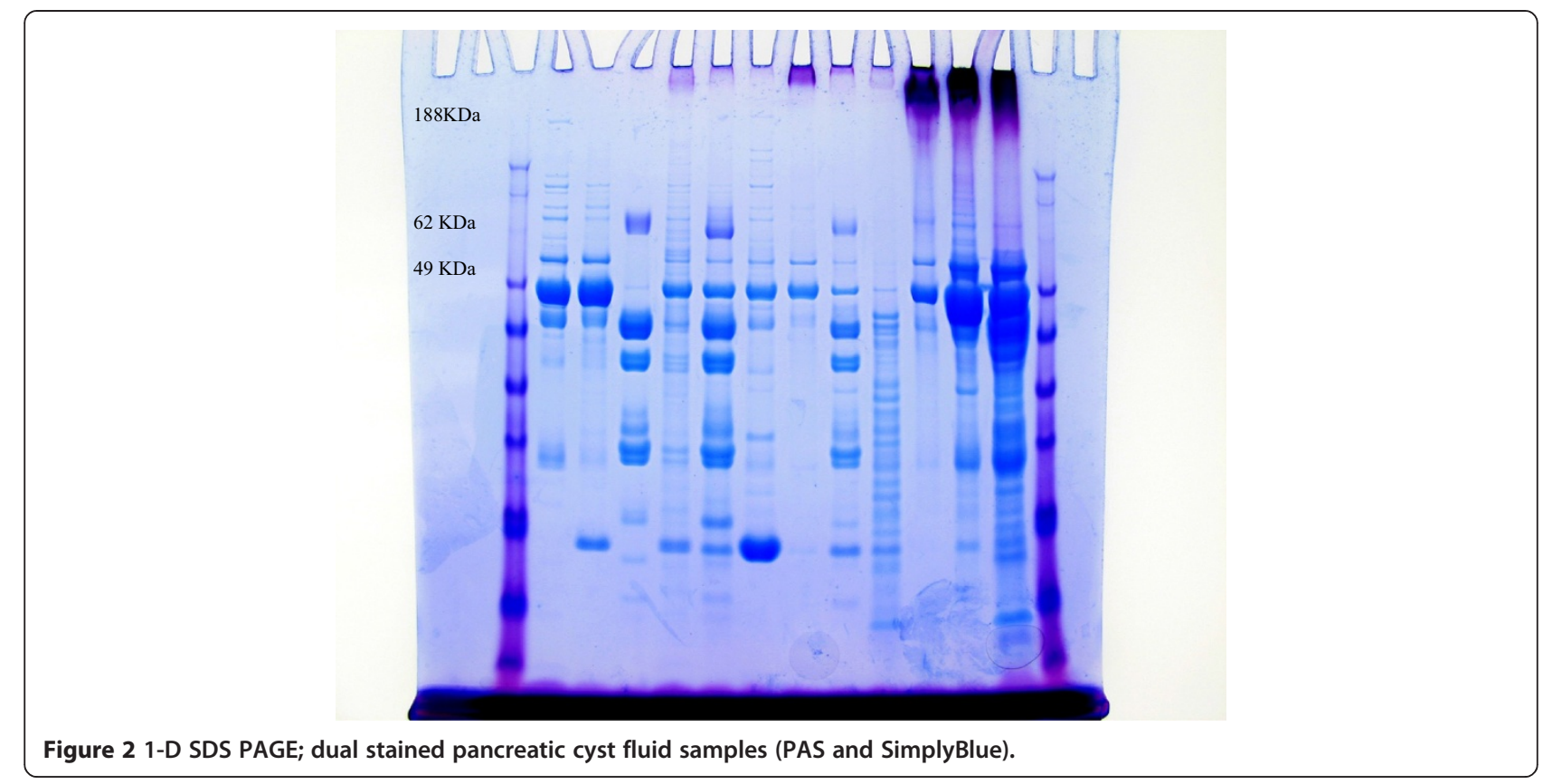

\section{Conclusion}

Pancreatic cyst fluid, which often shows a high degree of variation from one sample to the next, is difficult to analyze. The technique of one dimensional SDS polyacrylamide gel electrophoresis of the pancreatic cyst fluid, followed by dual staining for mucin (PAS) and protein (Simply Blue Safestain), provides a means of concentrating and visualizing the mucins. This allows the accurate differentiation of mucinous from non-mucinous histology in pancreatic cysts.

\section{Methods}

Samples: Between November 2005 and January 2011, twenty-eight fluid samples were obtained from patients with surgically excised cystic pancreatic lesions, under an IRB approved protocol for the purpose of this research. Each lesion had an unequivocal histological diagnosis on postoperative pathologic examination, as listed in Table 3. All IPMN samples categorized as benign displayed low- grade dysplasia by definition. Those cases of IPMN displaying high-grade dysplasia were categorized as malignant. Fluid samples were obtained by a single investigator (JMS) in the course of operative resection, either from the in situ specimen or immediately upon removal of the cyst from the patient. The fluid samples were frozen immediately within dry ice in plastic containers and then transferred directly to a minus 70 degree freezer at the conclusion of the operation. Pseudocysts were treated with enteric drainage, but a portion of the pseudocyst cyst wall was sent for histology, and all of these cysts resolved following treatment.

\section{Sample preparation}

Each sample was thawed and vortexed to ensure thorough mixing. An aliquot was removed and the sample refrozen. Protein content of the fluid was determined using the Pierce BCA protein assay kit (Thermo Scientific, Rockford IL), and 5 ug of protein used, as this is a commonly

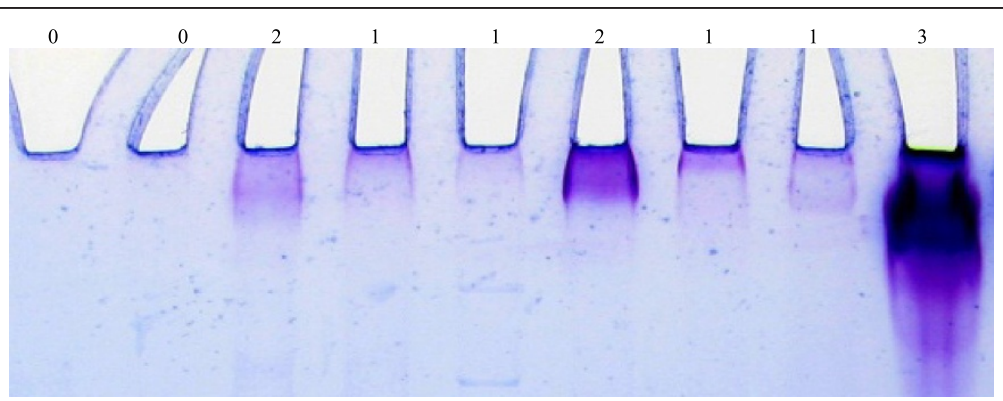

Figure 3 Close up of representative lane origins and the purple, dual stained bands used for scoring. Visual staining scores are above each specimen. 
employed amount to allow clear separation of protein bands during electrophoresis. In samples where the protein concentration was too low to reach the 5 ug level $(\mathrm{N}=4$ of 28$)$, the maximum volume for gel loading $(6.5 \mathrm{ul})$ was used.

\section{Electrophoresis}

Cyst fluid samples were run on a 4-12\% NuPage Novex Bis-tris minigels (Invitrogen, Carlsbad CA) with MES (2morpholinoethanesulfonic acid) running buffer containing $50 \mathrm{mM}$ MES, $50 \mathrm{mM}$ Tris Base, 0.1\% SDS, $1 \mathrm{mM}$ EDTA at $\mathrm{pH} 7.3$.

Samples were run under reducing conditions, prepared by combining the sample with 2.5 ul NuPage sample buffer $(4 \times)$, 1ul NuPage reducing agent $(10 \times)$, and then brought to a final volume of $10 \mathrm{ul}$. Samples were heated to 70 degrees $\mathrm{C}$ for 10 minutes (as per manufactures protocol), vortexed, spun down and loaded on the gel. The gel was then run at 200 volts constant for 35 minutes.

\section{Staining}

Primary staining was done with periodic acid-Schiff (PAS). Following electrophoresis the gel was immersed in $12.5 \%$ trichloracetic acid for 20 minutes, then 1\% periodic acid for 25 minutes in the dark. It was then immersed in Schiff's fuchsin-sulfite reagent (Sigma-Aldrich, St. Louis $\mathrm{MO})$ for 15 minutes in the dark, washed in $0.5 \%$ metabisulfate twice and then washed in water until the gel destained. The gel was then photographed. As seen in Figure 1, results showed red staining of the glycoproteins.

The secondary stain utilized the SimplyBlue SafeStain (Invitrogen, Carlsbad CA). Following PAS staining the gel was placed in $100 \mathrm{ml}$ of water and microwaved to almost boiling; the water was then discarded, with this process repeated twice. The gel was then covered with SimplyBlue SafeStain and microwaved about 1.5 minutes, washed in $100 \mathrm{ml}$ water and then $20 \mathrm{ml}$ of $20 \% \mathrm{NaCl}$ was added for 5 minutes. The gel was then photographed. The results showed blue staining of all proteins and purple (red primary and blue secondary) staining of glycoproteins (Figures 2 and 3).

\section{Glycoprotein stain scoring}

The dual stained gels were visually assessed by an evaluator blinded to the cyst fluid histology. The degree of mucin staining was accessed immediately downstream of the well. Staining at the 0 level was designated negative for mucin. Any detectable mucin staining was designated as positive and given $\mathrm{a}+1,+2$ or +3 valuation (Figures 2 and 3 ).

\section{Carcinoembryonic Antigen (CEA)}

The carcinoembryonic antigen (CEA) in each fluid sample was determined using an enzyme-linked immunosorbent assay (ELISA) kit for CEA (ALPCO, Salem NH) as per manufacturer's protocol.

\section{Statistical analysis}

Binomial distribution prediction intervals were calculated for the false positive and false negative rates using the technique of Krishnamoorthy [16].

Competing interests

The authors declare that they have no competing interests.

\section{Authors' contributions}

JS participated in study design, sample acquisition and data analysis, and preparation of the manuscript. MM was responsible for data acquisition, sample analysis, data analysis and preparation of the manuscript, and participated in study design. WS was responsible for data acquisition, sample analysis, data analysis and preparation of the manuscript, and participated in study design. KB participated in study conception and design, and manuscript preparation. JCD participated in study conception and design, and manuscript preparation. JLD participated in data analysis and statistical review. All authors read and approved the final manuscript.

\section{Acknowledgements}

This research was funded in part by grants from Essentia Health/Duluth Clinic and Whiteside Institute for Clinical Research.

\section{Author details}

${ }^{1}$ Departments of Surgery, Essentia Health System/Duluth Clinic; University of Minnesota Medical School, Duluth, MN, USA. ${ }^{2}$ Biomedical Sciences, Essentia Health System/Duluth Clinic; University of Minnesota Medical School, Duluth, MN, USA. ${ }^{3}$ Statistics, University of Wisconsin, Madison, WI, USA.

${ }^{4}$ Gastroenterology, Essentia Health System/Duluth Clinic; University of Minnesota Medical School, Duluth, MN, USA.

Received: 28 January 2014 Accepted: 4 November 2014 Published: 1 December 2014

References

1. Tanaka M, Chari S, Adsay V, Fernandez-del Castillo C, Falconi M, Shimizu M, Yamaguchi K, Yamao K, Matsuno S: International consensus guidelines for management of intraductal papillary mucinous neoplasms and mucinous cystic neoplasms of the pancreas. Pancreatology 2006, 6:17-32.

2. Khalid A, Brugge W: ACG practice guidelines for the diagnosis and management of neoplastic pancreatic cysts. Am J Gastroenterol 2007, 102:2339-2349.

3. Stelow EB, Shami VM, Abbott TE, Kahaleh M, Adams RB, Bauer TW, Debol SM, Abraham JM, Mallery S, Policarpio-Nicolas ML: The use of fine needle aspiration cytology for the distinction of pancreatic mucinous neoplasia. Am J Clin Path 2008, 129:67-74.

4. Michaels PJ, Brachtel EF, Bounds BC, Brugge WR: Bishop-Pitman M Intraductal papillary mucinous neoplasm of the pancreas: cytologic features predict histologic grade. Cancer 2006, 108(3):163-173.

5. Obeso EG, Murphy E, Brugge W, Deshpande V: Pseudocyst of the pancreas: the role of cytology and special stains for mucin. Cancer Cytopathol 2009, 117:101-107.

6. Morris-Stiff G, Lentz G, Chalikonda S, Johnson M, Biscotti C, Stevens T, Walsh RM: Pancreatic cyst aspiration analysis for cystic neoplasms: Mucin or carcinoembryonic antigen-Which is better? Surgery 2010, 148:638-645.

7. Compagno J, Oertel JE: Mucinous cystic neoplasms of the pancreas with overt and latent malignancy (cystadenocarcinoma and cystadenoma). American Society of Clinical Pathologists 1977, 69(6):573-580.

8. Ke E, Patel BB, Liu T, Li XM, Haluszka O, Hoffman JP, Ehya H, Young NA Watson JC, Weinberg DS, Nguyen MT, Cohen SJ, Meropol NJ, Litwin S, Tokar $\lrcorner$, Yeung AT: Proteomic analyses of pancreatic cyst fluids. Pancreas 2009, 38:33-42.

9. Ali M, Lillehoj EP, Park Y, Kyo Y, Chul Kim K: Analysis of the proteome of human airway epithelial secretions. Proteome Science 2011, 9:4.

10. Brugge WR, Lewandrowski K, Lee-Lewandrowski E, Centeno BA, Szydlo T, Regan S, Fernandez del Castillo C, Warshaw AL: Diagnosis of pancreatic cystic neoplasms: a report of the cooperative pancreatic cyst study gastroenterology. Gastroenterol 2004, 126:1330-1336. 
11. Ryu JK, Woo SM, Hwang JH, Jeong JB, Yoon YB, Park IA, Han JK, Kim YT: Cyst fluid analysis for the differential diagnosis of pancreatic cysts. Diagn Cytopthol 2004, 31:100-105.

12. Lewandrowski KB, Southern JF, Pins MR, Compton CC, Warshaw AL: Cyst fluid analysis in the differential diagnosis of pancreatic cysts. Ann Surg 1993, 217:41-47.

13. Allen PJ, Qin L, Tang L, Klimstra D, Brennan MF, Lokshin A: Pancreatic cyst fluid protein expression profiling for discriminating between serous cystadenoma and intraductal papillary mucinous neoplasm. Ann Surg 2009, 250:754-760.

14. Haab BB, Porter A, Yue T, Li L, Scheiman J, Anderson MA, Barnes D, Schmidt $M$, Feng Z, Simeone DM: Glycosylation variants of mucins and ceacams as candidate biomarkers for the diagnosis of pancreatic cystic neoplasms. Ann Surg 2010, 251:937-945.

15. Tian Y, Zhang H: Glycoproteomics and clinical applications. Proteomics Clin Appl 2010, 4:124-132.

16. Krishnamoorthy K, Peng J: Improved closed-form prediction intervals for binomial and poisson distributions. Fuel and Energy Abstracts 2011, 141(5):1709-1718.

doi:10.1186/1559-0275-11-42

Cite this article as: Streitz et al:: Differentiation of mucinous from non-mucinous pancreatic cyst fluid using dual-stained,

1 dimensional polyacrylamide gel electrophoresis. Clinical

Proteomics 2014 11:42.

\section{Submit your next manuscript to BioMed Central and take full advantage of:}

- Convenient online submission

- Thorough peer review

- No space constraints or color figure charges

- Immediate publication on acceptance

- Inclusion in PubMed, CAS, Scopus and Google Scholar

- Research which is freely available for redistribution 